\title{
Avances en la lucha contra la desigualdad y la violencia por razón de género en el ámbito laboral(*)
}

\author{
Advances in combating against inequality and gender violence in the \\ workplace
}

\author{
Sergio Quiñones Infante ${ }^{(*)}$ \\ Perú - Pontificia Universidad Católica del Perú \\ Claudia Tejada Yépez ${ }^{(* *)}$ \\ Perú - Estudio Barrios \& Fuentes Abogados
}

\begin{abstract}
Resumen: El objeto del presente artículo es evaluar los principales avances que el Estado peruano ha conseguido en la tarea de prevenir y erradicar la desigualdad y la violencia por razón de género en el ámbito laboral, habida cuenta que en nuestro país ambos fenómenos constituyen gravísimos problemas que deben ser combatidos de manera decidida y frontal. No es, pues, una tarea en la que nuestro país se encuentre solo; por el contrario, se trata de un reto global con el que todas y todos aspiramos a vivir en un mundo más igualitario y seguro. El gran objetivo en esta materia al 2021 debería ser el de llegar a ser un país bicentenario en el que las brechas salariales se hayan reducido sustancialmente, en el que las mujeres tengan plena igualdad de oportunidades y vivan libres de violencia, en el marco de la implementación de políticas públicas para promover la igualdad de género y el empoderamiento de todas las mujeres a todos los niveles.
\end{abstract}

Palabras clave: Derecho Laboral - Ámbito laboral - Igualdad de Género - Violencia de Género - Estado Peruano - Sistema Romano

\begin{abstract}
The purpose of this article is to assess the main progress that the peruvian state has made in preventing and eradicating gender-based inequality and violence in the workplace, given that in our country both phenomena constitute very serious problems that must be fought decisively and frontally. It is not, therefore, a task in which our country is alone; on the contrary, it is a global challenge with which we all aspire to live in a more equal and safer world. The big objective in this area by 2021 should be to become a bicentennial country in which wage gaps have been substantially narrowed, where women have full equal opportunities and live free from violence, within the framework of the implementation of public policies to promote gender equality and the empowerment of all women at all levels.
\end{abstract}

Key words: Labour Law - Workplace - Gender Equality - Gender-Based Violence Peruvian State - Roman System

$\left(^{*}\right) \quad$ Nota del Editor: este artículo fue recibido el 23 de octubre de 2019 y su publicación fue aprobada el 4 de noviembre de 2019

${ }^{* *}$ Magíster en Trabajo y Política Social por la Universidad Autónoma de Barcelona. Profesor de Derecho del Trabajo en la Pontificia Universidad Católica del Perú. Director General de Derechos Fundamentales y Seguridad y Salud en el Trabajo del MTPE. Miembro Extraordinario de la Asociación Civil lus et Veritas. Miembro extraordinario de lus et Veritas. Contacto: quinones.sa@pucp.edu.pe.

${ }^{* * *}$ ) Magíster en Desarrollo Organizacional y Dirección de Personas por la Universidad del Pacífico Business School. Abogada por la Pontificia Universidad Católica del Perú. Socia del Área Laboral del Estudio Barrios \& Fuentes Abogados. Contacto: ctejada@bafur.com.pe. 


\section{Introduc ción}

Según datos del Instituto Nacional de Estadística e Informática (INEI) al 2017, en nuestro país, el nivel de participación de las mujeres en la PEA es más baja que el de los hombres, $61 \%$ aproximadamente. Asimismo, la concentración en el empleo informal es más alta, siendo un $75 \%$ aproximadamente, y el nivel de subempleo femenino también lo es con un $54 \%$ aproximadamente.

En este contexto, resulta pertinente recordar que reducir las desigualdades y erradicar la violencia de género constituye un objetivo de política pública plenamente alineado con el mandato de nuestra Constitución Política, el Acuerdo Nacional y los Objetivos de Desarrollo Sostenible (ODS) de Naciones Unidas para el 2030.

En línea con ello, y en lo que concierne específicamente al mundo del trabajo, nuestro propósito es evaluar las recientes acciones que el Estado ha implementado en la tarea de reducir la brecha salarial por razón de género, que en el Perú asciende a $29 \%$ aproximadamente y que se ha mantenido en niveles similares en los últimos 10 años, así como para combatir todas las formas de hostigamiento sexual laboral.

Sobre lo primero, cabe recordar que mediante el Decreto Supremo 002-2018-TR se reglamentó la Ley 30709 - Ley que prohíbe la discriminación remunerativa entre hombres y mujeres, especificando el alcance de las obligaciones de los empleadores, fijando las reglas para la fiscalización de dichas obligaciones y estableciendo las herramientas que el Estado debe proveer a los empleadores para orientarles en el cumplimento de la Ley.

En esa línea, el Ministerio de Trabajo y Promoción del Empleo - MTPE aprobó la Guía que fija las pautas referenciales para que los empleadores puedan elaborar sus cuadros de categorías y funciones, y puedan diseñar sus planes y políticas de igualdad y, más recientemente, aprobó también la Guía Metodológica para la valorización objetiva de puestos de trabajo, que permite a los empleadores contar con una herramienta para valorar adecuadamente sus puestos de trabajo, asignarles igual remuneración a aquellos que tengan igual valor, y así coadyuvar a la erradicación de la discriminación remunerativa entre hombres y mujeres a nivel nacional.

En cuanto a lo segundo, hay que destacar la aprobación del Decreto Legislativo 1410 que estableció nuevas tipificaciones que sancionan conductas de acoso sexual y modificó la Ley 27942 - Ley de Prevención y Sanción del Hostigamiento Sexual, explicitando que el hostigamiento es una forma de violencia contra la mujer. Posteriormente, a través del Decreto Supremo 014-2019-MIMP, se aprobó el Reglamento de la citada Ley.

Como consecuencia de los mandatos establecidos en el Reglamento en cuestión, el MTPE formalizó la creación y funcionamiento del Servicio "Trabaja Sin Acoso" para la atención y asesoría legal y psicológica de casos de hostigamiento sexual laboral, tanto telefónica como presencialmente.

\section{Información estadística relevante $^{(1)}$}

De manera previa al análisis concreto que efectuaremos acerca de los avances que el Estado peruano ha conseguido en la tarea de prevenir y erradicar la desigualdad y la violencia por razón de género en el ámbito laboral, consideramos indispensable referirnos a algunas cifras y datos estadísticos que nos muestren el panorama general de la situación de la mujer en el mundo del trabajo.

Para ello resulta pertinente remitirnos al profesor Harari (2017), para quien la jerarquía (desigualdad) por razón de género ha estado presente en todas las organizaciones sociales humanas, resultando transversal al tiempo y espacio. En virtud de dicha jerarquía, en prácticamente todos los momentos históricos y lugares del planeta, los hombres han mantenido una posición de ventaja sobre las mujeres. En nuestra opinión, esta "desigualdad de partida" que afecta y condiciona sustancialmente la incorporación del género femenino al mercado de trabajo se ha cimentado históricamente sobre la base de la división sexual del trabajo y del modelo de patriarcado.

De este modo se ha generado una marcada segregación ocupacional y condiciones de empleo precarias (incluyendo la brecha salarial) que impiden alcanzar una igualdad sustancial entre los géneros. Dicha desigualdad por razón de género, manifestada concretamente en el mundo del trabajo, es efectivamente extendida a nivel global, recurrente en el tiempo y contiene una serie de características transversales.

(1) El presente apartado se basa íntegramente en: Quiñones, S (2019). Género y Trabajo: La búsqueda de igualdad en el ámbito de las relaciones laborales. Lima: PUCP, pp. 47-65. 
En primer lugar, y tal como lo afirma la Organización Internacional del Trabajo (2018), la tasa mundial de participación femenina en el mercado laboral es 26.5 puntos porcentuales más baja que la de los hombres, y dicha inactividad económica femenina responde fundamentalmente a la necesidad de atender las responsabilidades derivadas del cuidado del hogar y de la dependencia.

En segundo lugar, la Organización Internacional del Trabajo alerta que las mujeres no solo tienen menos probabilidades de participar en el mercado de trabajo, sino que las que sí lo hacen tienen menos probabilidades de encontrar un empleo. De hecho, para el 2018, por ejemplo, la tasa global de desempleo femenino, que fue del $6 \%$, superó a la masculina en aproximadamente 1 punto porcentual.

En tercer, y último lugar, destaca el estudio de la Organización Internacional del Trabajo, que muestra que la brecha global de participación económica sigue siendo amplísima, lo que se refleja en que la brecha de ingresos y salarios en perjuicio de las mujeres constituya todavía hoy un enorme problema que urge atención inmediata, dado que las mujeres ganan, en promedio, $20 \%$ menos que los hombres. Esto se debería a su presencia mayoritaria en sectores y empleos de bajos ingresos, pero también a la persistencia específica de una brecha salarial por razón de género.

La situación en nuestro país no difiere mucho del panorama global antes referido. Así, según datos del INEI (2), en el Perú la tasa de actividad femenina, o de participación laboral, para el año 2017 fue de aproximadamente 64\% respecto del total de mujeres y adolescentes en edad de trabajar. Dicha tasa de actividad fue 17 puntos porcentuales inferior a la de participación de los hombres en el mercado laboral, que ascendió a $81 \%$ para el mismo año. En lo que respecta a la tasa de subempleo, tenemos que, para el año 2017 , el $56,1 \%$ del total de las aproximadamente, 7 millones 300 mil mujeres ocupadas en alguna actividad económica lo hacían subempleadas por horas o por ingresos, mientras que solo el $43,9 \%$ estaban adecuadamente empleadas u ocupadas. La tasa de subempleo femenina superó en alrededor de 20 puntos porcentuales a la masculina, que ascendió al 35,6\% para el 2017.

Por último, un tercer indicador de suma relevancia es el relativo a la brecha de ingresos por razón de género. Sobre el particular, habíamos adelantado ya que la brecha de ingresos entre hombres y mujeres en el Perú asciende aproximadamente a $29 \%$. Dicho porcentaje resulta de la diferencia entre el "ingreso laboral promedio mensual" de los hombres (S/ 1566 soles) y el de las mujeres (S/ 1107 soles), según los datos de la Encuesta Nacional de Hogares sobre Condiciones de Vida y Pobreza 2017, elaborada por el INEI ${ }^{(3)}$. Importante advertir que este "escenario de desigualdad" en el mundo del trabajo, que desfavorece sustancialmente al género femenino, debe ser necesariamente complementado con otros factores que profundizan aún más las brechas de género.

Por un lado, debemos subrayar que no se trata solo de desigualdad en el acceso al empleo o en la retribución, sino también que las mujeres enfrentan con frecuencia el acoso sexual en el lugar de trabajo. En efecto, "como el acoso es una violencia basada en el sexo y género del agresor y de la víctima, el acoso sexual caracteriza una conducta de discriminación por género" (Reis De Araujo, 2018, pág. 17).

En esa misma línea, la autora citada refiere que una situación que se asemeja al acoso y que influye negativamente el ambiente laboral "consiste en que la empresa exige que las trabajadoras mujeres adopten una vestimenta provocativa para atraer a los clientes y generar mayores ganancias a la empresa (...) [lo que] además de caracterizar la violación a la intimidad personal y al derecho a la propia imagen de la trabajadora, puede, también, estimular la práctica del acoso sexual laboral ambiental" (2018, pág. 17).

Por otro lado, no podemos dejar de llamar la atención sobre los mayores niveles de vulnerabilidad dentro del propio grupo vulnerable. Así, por ejemplo, las más de 370 mil trabajadoras del hogar que laboran en el Perú requerirían medidas de atención y de protección específicas en atención al mayor nivel de exposición a malas condiciones de trabajo o violencia en el que se encuentran dentro de los domicilios particulares en los que prestan servicios.

Otro caso que también ameritaría una atención específica es el de la población femenina indígena. Como señala Del Águila (2015), el porcentaje de la población total de mujeres y hombres mayores de catorce años que se autoidentificó como perteneciente a un pueblo indígena ascendía a $15,5 \%$, según la Encuesta Nacional de Hogares del año 2014. En la citada investigación se indica, por ejemplo, que existía una brecha significativa

(2) Fuente: Instituto Nacional de Estadística e Informática - Encuesta Nacional de Hogares sobre Condiciones de Vida y Pobreza 2017.

(3) Fuente: Instituto Nacional de Estadística e Informática - Encuesta Nacional de Hogares sobre Condiciones de Vida y Pobreza 2017. 
entre los salarios de mujeres indígenas y afrodescendientes y el de blancas o mestizas, de tal suerte que el salario de las primeras fluctuaba entre $\mathrm{S} / 587$ soles para mujeres indígenas de la Amazonía y S/ 595 soles para mujeres aimaras; mientras que, en el segundo grupo, el salario variaba entre S/ 919 soles para las autoidentificadas como mujeres blancas y S/ 944 soles para las mujeres mestizas. Se trata de diferencias de ingresos que "muestran claramente la interseccionalidad o doble discriminación de las mujeres indígenas en el ámbito laboral" (Del Águila, 2015, pág. 81).

Precisamente, Hugo Ñopo (2012) destaca que, pese a que la tasa de actividad promedio de la población indígena en la región latinoamericana es de $63-69 \%$, las personas indígenas están mayoritariamente concentradas en el trabajo por cuenta propia y en el sector agrícola, con ingresos que resultan significativamente más bajos que los de sus pares no indígenas.

Más aún, "la interacción de etnicidad y género es de crucial importancia (...) a pesar de los aumentos en la participación y los ingresos de las mujeres en la fuerza laboral, las mujeres indígenas permanecen persistentemente en el fondo de la distribución de los ingresos, mostrando los niveles más altos de pobreza y exclusión" (Ñopo, 2012, pág. 247). En suma, Ñopo concluye que, a pesar de las importantes mejoras en materia de empleo producidas en las últimas décadas en la región, las mujeres y las minorías étnicas (pueblos indígenas y afrodescendientes) continúan sustancialmente marginados en los mercados laborales.

\section{Medidas implementadas para reducir la brecha salarial}

La actual "Política General de Gobierno al 2021", aprobada por Decreto Supremo 056-2018-PCM, señala como uno de sus cinco ejes el "desarrollo social y bienestar de la población" (eje 4), dentro del cual se reconoce como uno de los lineamientos prioritarios "promover la igualdad y no discriminación entre hombres y mujeres" (lineamiento 4.6).

Por su parte, el Acuerdo Nacional(4) prevé dentro de su segundo objetivo general, vinculado al desarrollo con equidad y justicia social, la Política de Estado 11, consistente en la "promoción de la igualdad de oportunidades sin discriminación". En virtud de dicha política, los actores políticos y sociales se comprometieron a dar prioridad efectiva a la promoción de la igualdad de oportunidades en favor de colectivos sistemáticamente discriminados (incluido, ciertamente, el de las mujeres), y para ello reconocen la necesidad de implementar temporalmente acciones afirmativas por parte del Estado, orientadas a garantizar la igualdad real de oportunidades económicas, sociales y políticas para toda la población.
A tal efecto, el Estado asumió (entre otros) los siguientes compromisos: combatir toda forma de discriminación, promoviendo la igualdad de oportunidades; fortalecer la participación de las mujeres en el Estado y la sociedad civil y, garantizar un acceso equitativo a las mujeres a recursos productivos y al empleo. En suma, desde el ámbito nacional, resulta claro que la reducción de la brecha salarial por razón de género, en tanto objetivo inherente a la igualdad de oportunidades entre mujeres y hombres, es una política de Estado plenamente vigente, $y$ constituye, además, una política nacional que forma parte integrante de la política general del gobierno actual.

Complementando lo anterior, debemos destacar que el Perú ha ratificado e incorporado a su ordenamiento jurídico interno como de obligatorio cumplimiento los cuatro convenios fundamentales de la OIT en torno a los cuales se articula el objetivo de lograr la igualdad por razón de género en el empleo: (i) el Convenio sobre igualdad de remuneración, 1951 (núm. 100) en el año 1960; (ii) el Convenio sobre la discriminación (empleo y ocupación), 1958 (núm. 111) en el año 1970; (iii) el Convenio sobre los trabajadores con responsabilidades familiares, 1981 (núm. 156) en el año 1986; y, (iv) el Convenio sobre la protección de la maternidad, 2000 (núm. 183), más recientemente, en mayo de 2016.

En conclusión, el Perú ha asumido también un conjunto de compromisos internacionales que ubican a la igualdad de oportunidades por razón de género y a la reducción de la brecha salarial como temas prioritarios de la agenda global. A nivel de normativa interna, la discriminación remunerativa ha sido reconocida como una manifestación de la violencia de género; en particular, la Ley 30364 - Ley para Prevenir, Sancionar y Erradicar la Violencia contra las Mujeres y los Integrantes del Grupo Familiar, reconoce la diferencia salarial por motivos de género como una manifestación de la violencia contra las mujeres.

Esta manifestación de violencia contra las mujeres ha sido definida como una situación

(4) Para mayor información, consultar: http://acuerdonacional.pe/ 
que se produce cuando se establecen diferencias salariales o remunerativas basadas en el sexo de la persona y sin criterios objetivos, la que puede ser directa o indirecta (artículo 2 del Decreto Supremo 002-2018-TR, Reglamento de la Ley 30709).

Tal como fue adelantado en el apartado introductorio, el Reglamento en cuestión ha especificado el alcance de las obligaciones de los empleadores, fijando las reglas para la fiscalización de dichas obligaciones por parte de la Superintendencia Nacional de Fiscalización Laboral SUNAFIL, y ha establecido las herramientas que el Estado debe proveer a los empleadores para orientarles en el cumplimento de la Ley 30709.

En cumplimiento a dicho mandato, el MTPE aprobó, mediante Resolución Ministerial 243-2018-TR, una primera guía que fija las pautas referenciales para que los empleadores puedan elaborar sus cuadros de categorías y funciones y puedan diseñar sus planes y políticas de igualdad; y, posteriormente, mediante Resolución Ministerial 145-2019-TR aprobó también la guía metodológica para la valorización objetiva de puestos de trabajo, que permite a los empleadores contar con una herramienta para valorar adecuadamente sus puestos de trabajo, asignarles igual remuneración a aquellos que tengan igual valor y así coadyuvar a la erradicación de la discriminación remunerativa entre hombres y mujeres a nivel nacional.

Esta segunda guía contiene una descripción mucho más detallada de todas las etapas que los empleadores deben seguir para valorar sus puestos de trabajo y elaborar sus cuadros de categorías y funciones, en concordancia con los criterios establecidos en la Ley 30709 y su Reglamento. Cabe destacar que, al igual que con la primera guía, esta propuesta metodológica se inspira en el modelo impulsado por la OIT, el cual incide en una valoración objetiva de puestos, evitando algún tipo de sesgos de género dentro de dicho proceso.

La guía metodológica para la valorización objetiva, sin discriminación de género, de puestos de trabajo y elaboración de cuadros de categorías y funciones está dirigida a las empresas del sector privado de todo rubro y tamaño, para que creen puestos de trabajo en condiciones de igualdad y sin discriminación hacia las mujeres, en caso sean empresas nuevas; y/o evalúen, reflexionen y, de ser necesario, modifiquen sus perfiles de puestos para reestablecer las condiciones de igualdad sin discriminación hacia las mujeres.

En ese sentido, la guía establece los requisitos para formar un equipo paritario y conocedor de la empresa, así como las funciones que este comité cumplirá (gestionar todo el proceso de valorización de puestos) y a cargo de quién está nombrarlo (la oficina de recursos humanos o la que haga sus veces). Los pasos concretos para la valorización objetiva de puestos son los siguientes:

a) Identificar los puestos de trabajo: recopilar los puestos actuales en una base de datos, para lo cual se aplicarán una serie de criterios que permitirán una clasificación eficiente.

b) Determinar el género de los puestos: a cada puesto de trabajo se le asignará un género, ya sea masculino o femenino. Para ello también se aplicarán tres criterios que permitirán definir el género asociado al puesto de trabajo: criterio social, criterio histórico y criterio de la propia planilla.

c) Valorizar los puestos de trabajo: una vez que se han realizado los pasos previos, se plantea que se proceda a determinar el puntaje de los puestos, esto es, hallar su valor relativo. Para ello, se deben seleccionar ciertos sub factores que permitan comparar, de manera general, todos los puestos de trabajo. Los sub factores deben ser divididos en niveles, según su frecuencia, intensidad, entre otras variables. Seguidamente, se debe recoger información sobre los puestos de trabajo para que, con dichos datos, se pueda asignar los niveles requeridos por cada sub factor, según la información de los puestos. Este proceso permitirá hallar el valor de los puestos.

d) Comparar los puestos y calcular las brechas existentes: una vez que se ha realizado la valoración de los puestos de trabajo, se propone que se proceda a la comparación de los puestos de trabajo para determinar las posibles brechas existentes. Ahí donde los puestos tengan el mismo valor, pero distinta remuneración, se deberá determinar si existen criterios objetivos que justifican tal distinción. En caso estos no se presenten, estaremos ante un posible supuesto de discriminación que deberá ser corregido.

e) Implementar medidas para eliminar la brecha salarial: una vez que se han identificado las brechas, se proponen ciertas pautas para poder corregir tal distorsión y que las remuneraciones de los trabajadores y trabajadoras puedan responder a criterios objetivos y de equidad.

Por otro lado, resulta pertinente destacar que, para garantizar el cumplimiento de las obligaciones contenidas en la Ley 30709 y su Reglamento, la SUNAFIL publicó el 
Protocolo 004-2019-SUNAFIL/INII, el cual tiene por objeto establecer las pautas para que la inspección del trabajo fiscalice adecuadamente las obligaciones contenidas en la normativa bajo comentario.

A ello se debe agregar que, en materia de discriminación remunerativa, la SUNAFIL ha realizado durante el año 2019 un total de 129 actuaciones de fiscalización a nivel nacional (105 de ellas por denuncia y 24 a través de operativos programados), 32 acciones de orientación y, en el marco de las campañas de difusión y conferencias informativas, se ha logrado informar y sensibilizar a más de dos mil ciudadanos y ciudadanas en materia de igualdad salarial y discriminación remunerativa a nivel nacional(5).

Finalmente, resta añadir a este conjunto de medidas la creación y funcionamiento de la "Mesa de Trabajo Tripartita que contribuya a promover y fortalecer la participación de las mujeres y la vigencia de sus derechos en el ámbito socio laboral", en el marco de lo establecido en la Resolución Ministerial 0732019-TR. La mesa tiene por objeto promover y fortalecer la participación de las mujeres y la vigencia de sus derechos en el ámbito socio laboral, coadyuvando a la mejora de la inserción y permanencia en el trabajo y de sus condiciones de empleo.

Dentro de sus principales funciones se prevé que tenga a su cargo realizar un diagnóstico sobre la problemática de las mujeres en el acceso, permanencia y salida del mercado laboral; así como su participación en los espacios de representación gremiales y sindicales; y, proponer medidas para promover la igualdad y mejorar las condiciones laborales de las mujeres, la conciliación de la vida laboral y familiar, y las responsabilidades familiares compartidas, en el marco de los instrumentos internacionales ratificados por el Estado peruano.

\section{Avances para prevenir y erradicar la violencia de género en el ámbito laboral}

La violencia de género en el trabajo es concebida como la realización o la amenaza de comportamientos y prácticas inaceptables, ya sea que se manifiesten una sola vez o de forma repetida, que tengan por objeto causar un daño físico, psicológico, sexual o económico, las cuales se motivan en el género o sexo de alguien o afectan desproporcionadamente a colectivos de un género o sexo determinado.

En ese sentido, este tipo de violencia se constituye como un grave problema social que se manifiesta en diversas formas: violencia psicológica, violencia sexual, violencia física y su expresión más extrema, los feminicidios. Asimismo, hay otras formas de violencia (profundamente arraigadas en nuestra sociedad) que son cotidianas y cuentan con tolerancia social, como es el caso del acoso en sus diversas modalidades, siendo uno de ellos el hostigamiento sexual laboral, o la ya comentada discriminación salarial entre hombres y mujeres.

El hostigamiento sexual laboral es definido como una forma de violencia que se expresa, a través de conductas de naturaleza o connotación sexual o sexista, no deseadas por la persona que las recibe, que pueden causar un clima intimidatorio, hostil o humillante, así como afectar a la víctima en su situación laboral. La importancia de prevenir y detectar el hostigamiento sexual de manera temprana reside en que, ante uno de estos casos, la integridad de la víctima es afectada, y es muy probable que, en tanto situación de violencia, esta pueda continuar escalando, poniendo en peligro la dignidad y seguridad de la víctima. Ahora bien, las acciones que el sector trabajo y promoción del empleo ha implementado en esta materia puedan agruparse en dos líneas de servicio principales.

De un lado, la creación formal del Servicio de Orientación y Acompañamiento para casos de hostigamiento sexual en el trabajo - Servicio "Trabaja Sin Acoso", a nivel de Lima Metropolitana, a través del Decreto Supremo 014-2019-TR. Y, en línea con lo anterior, la aprobación de los "Lineamientos del servicio de orientación y acompañamiento para los casos de hostigamiento sexual en el trabajo Trabaja Sin Acoso", a través de la Resolución Ministerial 222-2019-TR. Sobre el particular, cabe destacar que en el transcurso de un año (octubre 2018 - octubre 2019), el Servicio "Trabaja Sin Acoso" ha atendido un total de 1,821 casos de hostigamiento sexual laboral y otros tipos de hostilidad y violencia laboral.

De estos casos, 1493 fueron atendidos a través de la "Línea Telefónica 1819", y 328 fueron atendidos de manera presencial a través de la Oficina "Trabaja Sin Acoso", ubicada en el primer piso de la sede del MTPE. EI 95\% de los casos atendidos específicamente en materia de hostigamiento sexual laboral fue reportado por mujeres, y en el $99 \%$ de esos casos reportados los agresores denunciados fueron hombres. El $77 \%$ de los casos atendidos específicamente en materia de hostigamiento sexual laboral

(5) Fuente: Intendencia Nacional de Inteligencia Inspectiva de la SUNAFIL. 
corresponde a empresas del sector privado; mientras que el $23 \%$ de los casos corresponde a entidades públicas.

De otro lado, se encuentran las acciones de fiscalización y orientación en materia de hostigamiento sexual laboral llevadas a cabo por la SUNAFIL. Así, en lo que va del año 2019, la SUNAFIL ha realizado a nivel nacional un total de 109 actuaciones de fiscalización en materia de hostigamiento sexual laboral, 83 de ellas por denuncia y 26 a través de operativos programados.

Por su parte, a lo largo del año 2019, la SUNAFIL ha realizado a nivel nacional un total de 181 acciones de orientación en materia de hostigamiento sexual laboral y actos de hostilidad laboral; a lo que se suma las campañas de difusión y conferencias informativas que ha beneficiado a un total de 564 ciudadanos y ciudadanas en materia de hostigamiento sexual laboral. Para concluir, y complementando las dos principales líneas de actuación antes reseñadas, consideramos importante destacar que el MTPE aprobó recientemente la "Guía Práctica para la Prevención y Sanción del Hostigamiento Sexual en el Lugar de Trabajo en el sector privado y público" a través de la Resolución Ministerial 2232019-TR; e impulsó la creación del Servicio "Trabaja Sin Acoso" en cuatro gobiernos regionales adicionales a Lima Metropolitana: Apurímac, Huánuco, Junín y Ucayali.

\section{El Convenio de la Organización Internacional del Trabajo sobre la eliminación de la violencia y el acoso en el mundo del trabajo}

El pasado 21 de junio del año 2019 se llevó a cabo la sesión plenaria de la 108 Conferencia Internacional de Trabajo de la Organización Internacional del Trabajo en la ciudad de Ginebra, Confederación Suiza, en la cual se aprobó la adopción del Convenio 190 sobre la eliminación de la violencia y el acoso en el mundo del trabajo y de la Recomendación 206 sobre la eliminación de la violencia y el acoso en el mundo del trabajo. Como parte del intenso debate tripartito que condujo a dicha aprobación, se reconoció que a nivel global existen expresiones diversas de violencia y acoso en la sociedad que se replican en el ámbito laboral; y que, frente a dichas expresiones, la respuesta ha sido insuficiente y desarticulada de parte de los actores involucrados.

Efectivamente, en el mundo, cada vez más, se incrementaron los casos de violencia en el ámbito laboral; no obstante, esta problemática no se encontraba visibilizada adecuadamente en los instrumentos nacionales e internacionales vinculados a los derechos laborales. En atención a ello, y a través del Convenio 190, la Organización Internacional del Trabajo establece que la violencia y el acoso laboral pueden llegar a ser una vulneración grave de los derechos humanos y una amenaza para el ejercicio del derecho a la igualdad de oportunidades, el derecho a un trabajo decente y el derecho a la dignidad.
La Organización Internacional del Trabajo también establece que la violencia y el acoso afectan la calidad de los servicios públicos y privados, la organización y las relaciones en el lugar de trabajo; y, sobre todo, perjudican gravemente la salud física, psicológica y sexual de las personas, en específico en los casos de violencia y acoso en el trabajo con motivo de género. El Convenio 190 define la violencia y el acoso como aquellos comportamientos y prácticas inaceptables, o la amenaza de la realización de dichos comportamientos y prácticas, que tengan por objeto, que causen o sean susceptibles de causar, un daño físico, psicológico, sexual o económico ya sea que se manifiesten una sola vez o de manera repetida. Este concepto incluye, entre otros, la violencia y el acoso por razón de género.

De esta forma, la violencia y el acoso por razón de género se configura cuando la violencia o acoso va dirigida contra las personas por razón de su sexo o género; o que afectan de manera desproporcionada a personas de un sexo o género determinado. Nótese que el concepto incluye, entre otros, el acoso y hostigamiento sexual laboral. En suma, conforme a la normativa internacional recientemente aprobada, queda claro que el acoso u hostigamiento sexual laboral constituye una de las diversas manifestaciones en las que la violencia en el trabajo se puede presentar.

En cuanto al ámbito de aplicación subjetiva, cabe destacar que el Convenio 190 resulta de aplicación obligatoria para todo tipo de "relación laboral", comprendiendo en ella a los trabajadores asalariados, con cualquier situación contractual, personas en formación, trabajadores despedidos, voluntarios, personas en busca de empleo y autoridades que ejercen como empleador. Se protege también a los implicados tanto del sector público como privado, a quienes ejercen en el sector formal e informal y en zonas urbanas y rurales. Desde el punto de vista objetivo, el Convenio 190 es aplicable a los casos de violencia y acoso laboral que ocurran durante el trabajo, en escenarios que tengan relación con el trabajo o que sean resultado de éste.

Cabe precisar que los espacios que se incluyen en dicho ámbito de aplicación 
objetivo comprenden los espacios públicos y privados del centro de trabajo; los lugares donde se les paga, descansan o comen los trabajadores; los viajes, eventos o actividades sociales organizadas por y para trabajo; las comunicaciones realizadas por trabajo a través de medios ordinarios y tecnologías; y los trayectos del domicilio al lugar de trabajo.

Por otro lado, algunas de las obligaciones y responsabilidades que el Convenio 190 prevé para los empleadores y que (en nuestra opinión) resulta pertinente resaltar, son las siguientes:

a) Generar una política del lugar de trabajo y un plan de acción ante casos de violencia y acoso laboral. Hacer obligatorio que se considere la gestión de seguridad y salud como respuesta ante las consecuencias de estos casos.

b) Identificar y proporcionar a los trabajadores y autoridades información sobre los peligros, riesgos y consecuencias sobrevinientes a los casos de violencia y acoso en el espacio de trabajo de su vigilancia.

c) Hacer visibles los casos de violencia y acoso e identificar los contextos en los que existe mayor riesgo de cometerse actos de violencia y acoso, con especial enfoque en los casos con motivo de género.

Por el lado del Estado peruano, el Convenio 190 señala que los estados miembros deberán, una vez ratificado el instrumento internacional, revisar y realizar el seguimiento respectivo al cumplimiento de las políticas y legislación aplicables a los casos de violencia y acoso laboral. Asimismo, deberá identificar situaciones laborales, amparadas en la diversidad de situaciones que indica el Convenio, que estén más expuestas a la violencia y el acoso laboral, a fin de que en ellas se refuerce la fiscalización de la regulación y sanciones a los y las involucrados.

Adicionalmente, los estados miembro asumirían la obligación de facilitar el acceso a mecanismos de solución de conflictos respecto a casos de violencia y acoso laboral; hacer efectiva la protección de la estabilidad laboral de las y los implicados antes, durante y después de la resolución de los casos; así como brindar a las víctimas de casos de violencia y acoso con motivo de género el acceso efectivo a mecanismos de presentación de quejas y de solución de conflictos, asistencia, servicios y vías de recurso y reparación que tengan en cuenta las consideraciones de género y que sean seguros y eficaces.

Dicho esto, y a modo de conclusión del presente artículo, consideramos que a partir de la aprobación del Convenio número 190, el Perú tiene por delante algunos retos importantes; dentro de los que queremos destacar los siguientes: (i) impulsar el proceso de ratificación del Convenio 190 sobre la eliminación de la violencia y el acoso en el mundo del trabajo; (ii) consolidar el rol garantista del Estado para la protección de las víctimas de hostigamiento sexual laboral; (iii) generar conciencia colectiva en la comunidad acerca del hostigamiento sexual laboral como una forma de violencia de género; y (iv) reducir los niveles de hostigamiento sexual en el trabajo, a partir de la promoción de acciones que sensibilicen y nos comprometan a todas y todos.

\section{Referencias bibliográficas}

Del Águila, Alicia (2015). Estudio sobre la situación laboral de las mujeres indígenas en el Perú. Lima: Oficina de la OIT para los Países Andinos.

Harari, Yuval (2017). Sapiens. De animales a dioses: Una breve historia de la humanidad. Barcelona: Debate.

Neves, Javier (2009). Introducción al derecho del trabajo. Lima: Fondo Editorial PUCP.

Ñopo, Hugo (2012). New century, old disparities: gender and ethnic earnings gaps in Latin America and the Caribbean. Washington: InterAmerican Development Bank and World Bank.

Organización Internacional del Trabajo (OIT). (2009). Tendencias mundiales del empleo de las mujeres. Ginebra: Oficina Internacional del Trabajo.

(2018). Perspectivas sociales y del empleo en el mundo: Avance global sobre las tendencias del empleo femenino 2018. Ginebra: Oficina Internacional del Trabajo.

Quiñones, Sergio (2011). Planes de igualdad y conciliación de la vida laboral, familiar y personal: dimensiones que se abordan y posiciones de los actores. Memoria de Investigación para la obtención del Máster Europeo en Trabajo y Política Social por la Universidad Autónoma de Barcelona.

(2012). Enfoques para conciliar la vida laboral, familiar y personal desde la política pública. Ius et Veritas, 45, pág. 324-332. Recuperado de http://revistas.pucp.edu.pe/index.php/iusetveritas/ article/view/12006/12574

Reis de Araujo, Adriane. (2018). La igualdad de género en la relación laboral: La experiencia en el derecho brasileño. Análisis Laboral, 42(487), pág. 15-19. 